\title{
A Case of Locked Pelvis, With No Urogenital Or Vascular Injury
}

\author{
William Hau Wuei Huang*, Juzaily Fekry Leong, Rizal Abdul Rani and Nor Hamdan Mohd Yahya \\ Department of Orthopaedic and Traumatology, University Kebangsaan Malaysia, Malaysia
}

Received: April 09, 2018; Published: April 19, 2018

*Corresponding author: William Hau Wuei Huang, Department of Orthopaedic and Traumatology, University Kebangsaan Malaysia, Kuala Lumpur, Malaysia, Tel: 126630355; Email: William_hau@rocket.com

\begin{abstract}
Locked pubic symphysis or overlapping pubic symphysis occurs in traumatic lateral compression of the pelvic ring. The pubic bone is dislocated and overlaps behind the contralateral pubic bone. Neglected cases showed poor long-term outcomes resulting in constant pubic symphysis discomfort during ambulation and dyspareunia. We describe a locked pelvis without any urogenital injury and were reduced with simple manoeuvres.
\end{abstract}

\section{Introduction}

Pelvic injury due to medial compression forces are common and well recognised but lateral compression pelvic injury resulting in an intact pubic bone displaced against the contralateral side is rare. The mechanism of locked pelvis is proposed based on Eggers concept that first described this injury1. When the femur is being hyperextended and adducted or abducted, this causes lateral compression to thrust to the pelvis. Due to the tension of the iliofemoral ligament, the femoral head is being locked to the acetabulum. The pubic symphysis ligaments can be disrupted with further compression of the pelvis and resulting the pubic bone to be trapped anterior or posterior to the opposite intact pubic bone[1,2]. A few methods have been reported about the manner of reduction of these injuries, either closed or open reduction. One of which is by flexing, abducting and externally rotating the femur to use it as a lever to reduce the pubic symphysis, with simultaneous constant force being applied to the iliac crest to avoid fracturing the femur[3]. However, in this patient, we failed to reduce the pubic symphysis using the above method and proceeded with open reduction.

\section{Case Report}

The 31-year-old man involved in a motor vehicle accident where his motorcycle was hit from the right side by a car. Unfortunately, while trying to stand, both of his thigh and pelvis were run over by another car. On arrival to emergency department, he was conscious, and vitals were stable. He complained of severe pain over right side of chest and suprapubic region. Examination revealed bruises and tyre marks over the right hip and pelvic region. Chest spring was positive due to right rib fractures, but pelvic spring was negative. The patient complained of pain over the suprapubic region, but examination did not elicit suprapubic tenderness. Pelvic radiograph (Figure 1) showed overlapping pubic bones. CT scan (Figures $2 \& 3$ ) confirmed the right pubis was dislocated behind the left pubis and entrapped by the right pubic tubercle.

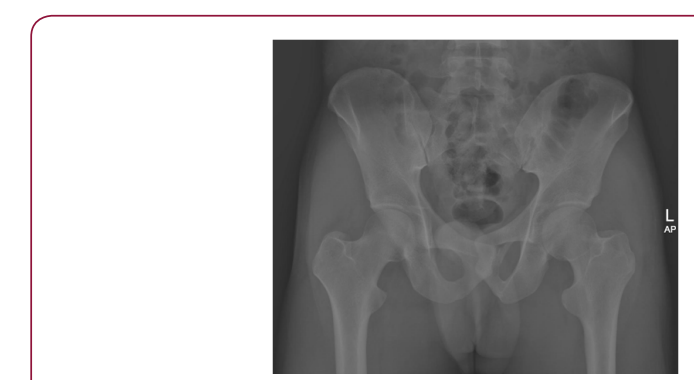

Figure 1: Right pubis dislocated behind the left pubis.

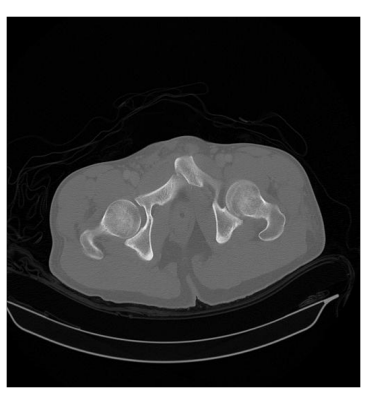

Figure 2: CT Scan shows overlapping of left pubic symphysis. 


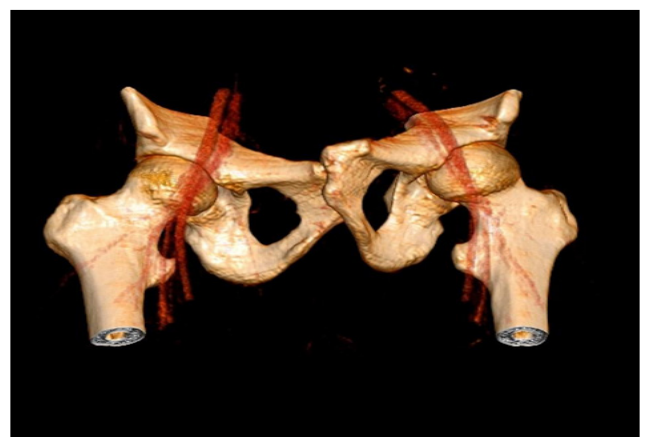

Figure 3: 3D Recon CT SCAN of the pelvis.

There were no other injuries noted.We attempted closed reduction of the pubic symphysis by using the femur as a lever, by flexing, abducting and applying an external rotation force on the femur as described by one case report[3]. However, we failed to reduce the pubic bone and proceeded with open reduction the following day.Open reduction was performed through a Pfannenstiel incision. The incision was made up to pubic rami. Vertical midline incision was made through the fascia. The pubic bone was exposed after the rectus muscles were elevated. We noted that the right pubis was dislocated behind the left pubis and entrapped by the right pubic tubercle. No other fracture was identified. Initially we attempted to reduce the right pubis by pulling the left pubis anteriorly to disengage it but failed. Subsequently, we used a blunt tip bone spike as a lever to disengage the posterior fragment from the anterior fragment. After the pubic have been disengaged, we were able to reduce the pubic symphysis and reduction was confirmed with image intensifier. In order to prevent recurrent dislocation of the pubic symphysis, we maintained the reduction with 6-hole recon plate (Figure 4).

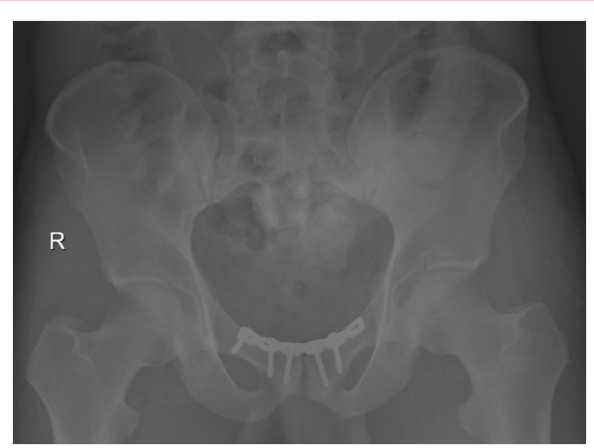

Figure 4: Post Op Pelvic X-ray.

Post operatively, patient recovered well. His urinary catheter remained clear since insertion from emergency department.He was able to ambulate well with wheelchair and was discharged 4 days after operation. Post operatively, the suprapubic pain resolved, and patient was advised against weight bearing for one month. At two weeks post-operative follow up, patient was able to ambulate independently. Despite the early ambulation against doctors advised, he only complained of mild groin pain especially during physical therapy session. Post operatively, patient recovered well.
His urinary catheter remained clear since insertion from emergency department.He was able to ambulate well with wheelchair and was discharged 4 days after operation. Post operatively, the suprapubic pain resolved, and patient was advised against weight bearing for one month. At two weeks post-operative follow up, patient was able to ambulate independently. Despite the early ambulation against doctors advised, he only complained of mild groin pain especially during physical therapy session.

\section{Discussion}

In cases with locked pelvis, it was found that anterior superior iliac spine had moved closer to the midline on the affected side compared to the opposite side. The condition occurs from a lateral compression force resulting in disruption of the stabilizing ligaments of the symphysis. The dislocation involves hyperextension, adduction and internal rotation, with posterior displacement of pubic bone. Closed reduction method involved locking the femur in flexion, abduction and external rotation and using the femur as a lever. However, not all locked pelvis can be reduced using closed methods. This condition is also associated with other concomitant injuries due to the forces required to dislocate the symphysis. It is mandatory to rule out bladder injury in every case, as the associated urogenital injuries have been reported up to $47 \%$ [4].All of the reported cases showed that patient either have urogenital injury or concomitant pelvic fracture. This patient had a urinary catheter inserted at the emergency department and the urine remained clear throughout the admission. Overlapped pubic symphysis reported cases all are male patients. According to Gamble et al, the cartilaginous disc of female pelvis compare to male has 2-3mm greater mobility[5]. In male the pubic symphysis is longer vertically. May be the likelihood of pubic bone overlapping is reduced in female is due to increase few millimetres of mobility.

\section{Conclusion}

Locked pelvis is rare. The patient may not complain of pain due to the "locking" nature of the injury therefore a high index of suspicion is required especially if the mechanism of trauma involves lateral compression of the pelvis. In patients with such pelvic injury, we must be suspicious of urogenital injury and vascular injuries.

\section{References}

1. Eggers GW (1952) Dislocations of the os coxae. Am J Surg 83(3): 300307.

2. Ansari S, Rollins J, Ebraheim NA (2003) Locked pubic symphysis with ipsilateral fracture neck of a femur. J Trauma 54(2): 376-378.

3. V Thulasiraman, Ramesh Pandian TR, S Ashok (2010) Locked pubic symphysis-A case series. Injury Extra 41: 20-24.

4. Alan T Blank, Mark Gage, Nirmal Tejwani, Toni McLaurin (2015) Overlapping Dislocation of the Pubic Symphysis with an Open Reduction and Anterior and posterior pelvic fixation A Case report. JBJS Case Connect 5: e6.

5. Gamble JG, Simmons SC, Freedman M (1986) The symphysis pubis: anatomic and pathologic considerations. Clin Orthop Relat Res 203: 261-272. 


\section{(C) (i) This work is licensed under Creative}

Submission Link: https://biomedres.us/submit-manuscript.php

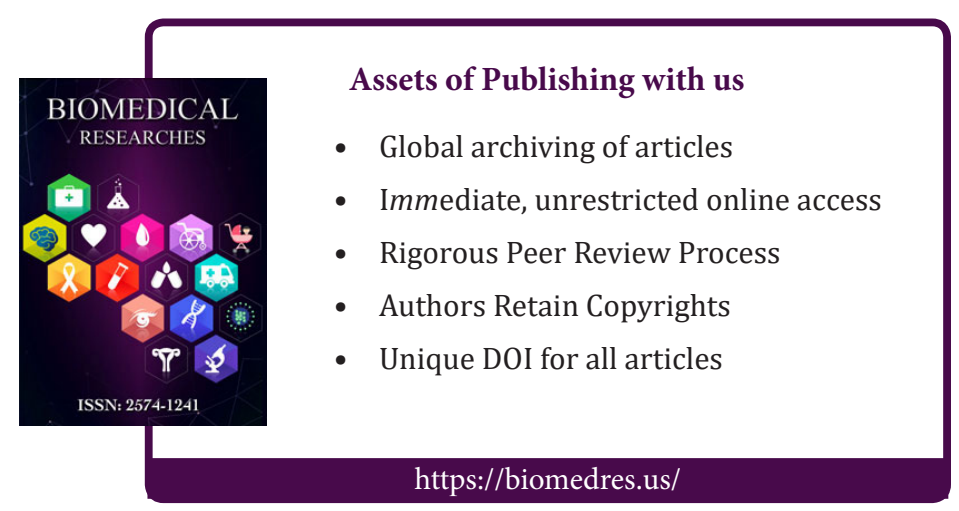

